\title{
A DIRECT-READING INSTRUMENT FOR MEASURING THE LOGARITHMIC DECREMENT AND WAVE LENGTH OF ELECTROMAGNETIC WAVES
}

\section{By Frederick A. Kolster}

\section{INTRODUCTION}

The laws of the United States governing radio communication specify, among other things, that at all stations the logarithmic decrement per complete oscillation in the wave trains emitted by the transmitter shall not exceed two-tenths, except when sending distress signals or messages relating thereto.

The importance of the regulation lies in the fact that when persistent oscillations of single frequency are emitted from a radio transmitting station much more selective receiving apparatus may be employed with advantage at receiving stations, permitting sharp tuning with consequent minimizing of interference caused by stations other than those with which communication is desired.

Since the logarithmic decrement is a measure of the decay of a train of waves, it is desirable that this decrement be made as small as possible in order that a series of decaying trains of waves may approach as near as possible to the condition of persistent oscillations. A wave train having a logarithmic decrement of two-tenths, the limit set by the Federal regulations, will have 24 complete oscillations before the amplitude of the last wave has decreased to I per cent of that of the first. Such a wave train is shown in Fig. I.

When full advantage is taken of the rapid scientific and technical progress which has been made in the methods of transmission of electromagnetic waves it is not at all difficult to comply with this requirement. In fact, it is practicable as well as desirable to keep well within this limiting value of two-tenths. 
The purpose of this paper is to describe a new direct-reading instrument for measuring the logarithmic decrement and wave length, especially designed about two years ago for the radio inspection service of the Bureau of Navigation, Department of Commerce, and since adopted by the War and Navy Departments.

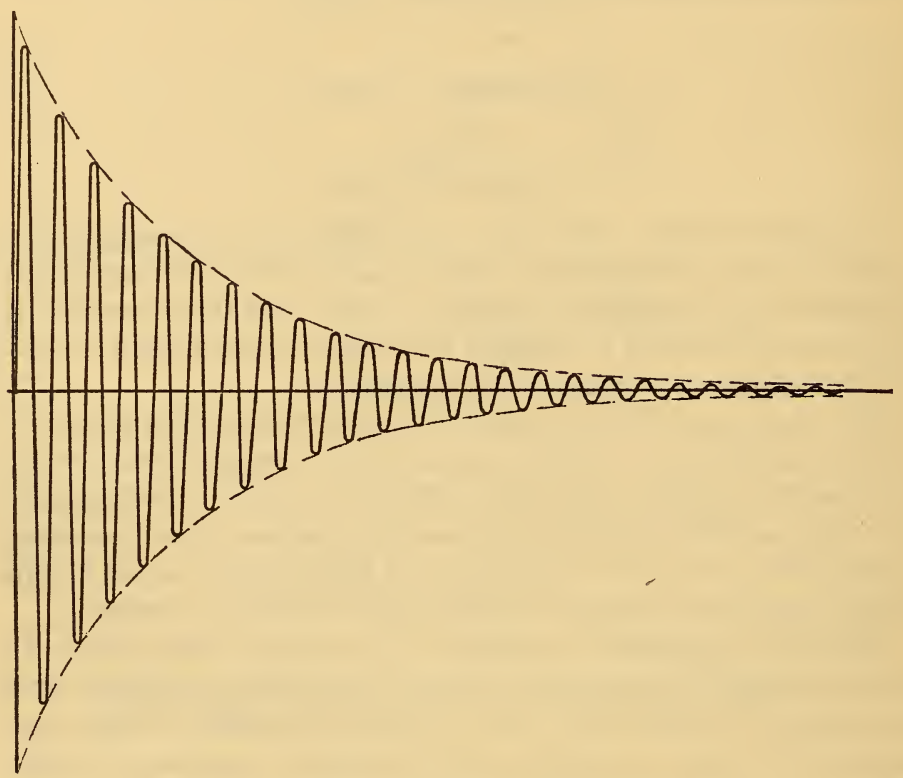

FIG. x.-Wave train with logarithmic decrement $=0.2$

\section{GENERAL THEORY}

When a condenser is discharged through inductance and resistance (Fig. 2), if the resistance is not too large, an oscillatory current is obtained, the value of which at any instant is given by the solution of the equation,

$$
L \frac{d i}{d t}+R i+\frac{\mathrm{I}}{C} \int i d t=\mathrm{o}
$$


The solution of this equation for the case of the circuit used in radio telegraphy takes the following form,

$$
i=C \omega V_{0} \epsilon^{-a t} \sin \omega t
$$

where $V_{0}$ is the initial potential difference across the condenser of capacity $C, \omega$ equals $2 \pi \times$ frequency, $\alpha$ equals $\frac{R}{2 L}$, and $\epsilon$ is the base of napierian logarithms.

Since $C \omega V_{0}$ is equal to $I_{0}$, the initial current amplitude, then

$$
i=I_{0} \epsilon^{-a t} \sin \omega t
$$

In Fig. $\mathrm{I}$ is shown an oscillatory current of this form and is such a current as would be produced in the circuit of Fig. 2. The rela-

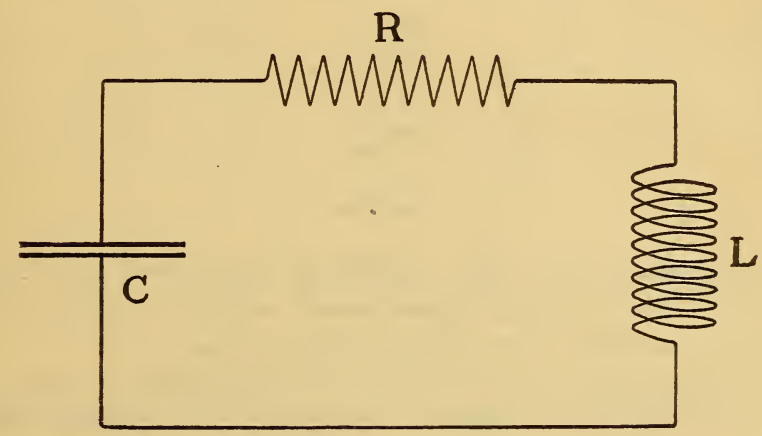

FIG. 2.- Oscillatory circuit containing inductance, capacity, and resistance

tion between any current amplitude, $I$ and that of the same sign preceding it, $I_{0}$, may be expressed as follows:

$$
I=I_{0} \epsilon^{-a \mathrm{~T}}
$$

$T$ is the period of one complete oscillation, and $\alpha$, which is equal to $\frac{R}{2 L}$, is the damping factor. $R$ and $L$ are the resistance and inductance, respectively, both measured at radio frequencies. 
The product of the damping factor and period of one complete oscillation, $\alpha T$, is called the logarithmic decrement and is commonly represented by $\delta$.

Hence,

and

$$
I=I_{0} \epsilon^{-\delta}
$$

$$
\delta=\log _{6} \frac{I_{0}}{I}
$$

The logarithmic decrement can also be written in terms of the constants of the circuit, for since $\alpha=\frac{R}{2 L}$

$$
\begin{aligned}
\delta & =\frac{R}{2 L} T \\
& =\frac{R}{2 L n} \\
& =\pi \frac{R}{L \omega} \\
& =\pi R C \omega
\end{aligned}
$$

The expression for the logarithmic decrement can also be derived by the following approximate method, which does not involve the solution of the differential equation:

The damping in a circuit is due to the dissipation of energy, and if we call $R$ the total equivalent resistance, including the effect of electromagnetic radiation, the energy dissipated in one period is $R i^{2} T$, where $i^{2}$ is the mean square current. In an undamped sine wave $i^{2}$ is $\frac{\mathrm{I}}{2} I_{0}{ }^{2}$. For the case shown in Fig. 3, we may put approximately,

$$
i^{2}=\frac{\mathrm{I}}{2} I_{1}^{2}=\frac{\mathrm{I}}{2} I_{0} I_{2}
$$

The total energy at the beginning of the period is $\frac{I}{2} L I_{0}{ }^{2}$ and at the end $\frac{I}{2} L I_{2}{ }^{2}$. The energy dissipated during one period is therefore the difference. 
By definition, the logarithmic decrement is

$$
\delta=\log \frac{I_{0}}{I_{2}} \text {, or } \frac{I_{0}}{I_{2}}=\epsilon^{8}
$$

hence

$$
\begin{gathered}
{ }^{2 \delta=} \log \frac{I_{0}{ }^{2}}{I_{2}{ }^{2}}=\log \frac{\frac{1}{2} L I_{0}{ }^{2}}{\frac{1}{2} L I_{2}{ }^{2}}=\log \left(\frac{\frac{1}{2} L I_{2}{ }^{2}+\frac{1}{2} R I_{0} I_{2} T}{\frac{1}{2} L I_{2}{ }^{2}}\right) \\
=\log \left(\mathrm{I}+\frac{R I_{0} T}{L I_{2}}\right)=\log \left(\mathrm{I}+\frac{R I_{0}}{L n I_{2}}\right)
\end{gathered}
$$

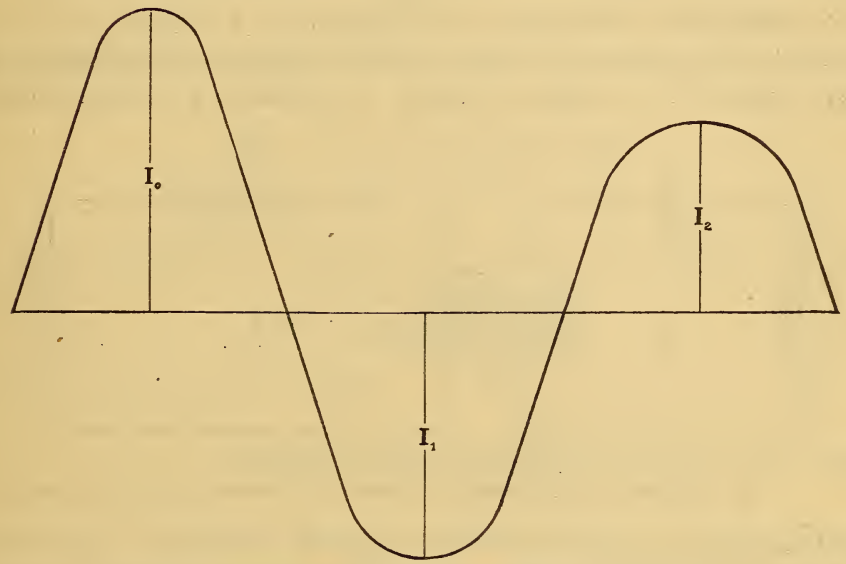

FIG. 3.-Oscillatory current with decaying amplitudes

therefore

$$
2 \delta=\log \left(\mathrm{I}+\frac{R}{L n} \cdot \epsilon^{\delta}\right)
$$

or

$$
\epsilon^{2 \hat{0}}=\mathrm{I}+\frac{R}{\mathrm{Ln}} \cdot \epsilon^{\delta}
$$

and

$$
\frac{\epsilon^{\delta}-\epsilon^{-\delta}}{2}=\frac{R}{2 L n}=\delta+\frac{\delta^{3}}{6}+\frac{\delta^{5}}{120}+\cdots \cdots
$$


If $\delta$ is small the second and succeeding terms are negligible and we have,

$$
\delta=\frac{R}{2 L n}
$$

This derivation is not as exact as the first which depended upon the solution of the differential equation, since the value assumed for the mean square of the current is not exact, but approximate. This method shows the relation between the logarithmic decrement and the dissipation of energy in an interesting manner.

If undamped oscillations are induced in a circuit, as by an alternator in a primary circuit loosely coupled to this circuit, we may derive in a simple manner a method of experimentally

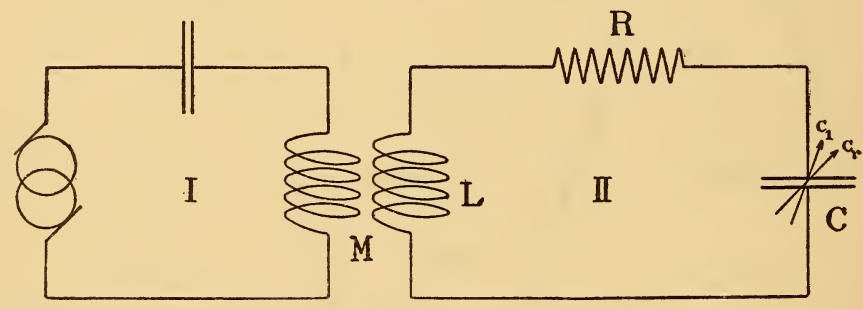

FIG. 4.-I. Exciting circuit producing undamped oscillations

II. Receiving circuit containing inductance, resistance, and variable capacity

determining the logarithmic decrement of the circuit. Circuit II in Fig. 4, which contains inductance, resistance, a variable capacity, and a current indicating device, is loosely coupled to the exciting circuit I in which there exist undamped oscillations, then at complete resonance, denoting by $I_{\mathrm{r}}$ the root mean square current,

$$
\text { (I) } I_{\mathrm{r}}=\frac{E}{R} \text { and (2) } L \omega-\frac{\mathrm{I}}{C_{\mathrm{r}} \omega}=\mathrm{o}
$$

Now, if circuit II is adjusted slightly out of resonance by changing the capacity from the resonant position $C_{\mathrm{r}}$ to another value $C$, then

$$
\text { (3) } I=\frac{E}{\sqrt{R^{2}+\left(L \omega-\frac{\mathrm{I}}{C \omega}\right)^{2}}} \text { and (4) } L \omega-\frac{\mathrm{I}}{C \omega}=\frac{\mathrm{I}}{C_{\mathrm{r}} \omega}-\frac{\mathrm{I}}{C \omega}
$$


$\frac{\mathrm{I}}{C_{\mathrm{r}} \omega}-\frac{\mathrm{I}}{\mathrm{C} \omega}$ representing a very small change in reactance, therefore,

Since

$$
\text { (5) } I=\frac{E}{\sqrt{R^{2}+\left(\frac{\mathrm{I}}{C_{\mathrm{r}} \omega}-\frac{\mathrm{I}}{C \omega}\right)^{2}}}=\frac{E}{\sqrt{R^{2}+\frac{\mathrm{I}}{C_{\mathrm{r}}^{2} \omega^{2}}\left(\frac{C_{\mathrm{r}}-C}{C}\right)^{2}}}
$$

$$
\begin{gathered}
\delta=\pi R C_{\mathrm{r}} \omega \\
\frac{\mathrm{I}}{C_{\mathrm{r}} \omega}=\frac{\pi R}{\delta}
\end{gathered}
$$

substituting this expression in equation (5), we have,

$$
\text { (6) } I=\frac{E}{\sqrt{R^{2}+\frac{\pi^{2} R^{2}}{\delta^{2}}\left(\frac{C_{\mathrm{r}}-C}{C}\right)^{2}}}
$$

Dividing equation (I) squared by equation (6) squared, we have

$$
\text { (7) } \frac{I_{\mathrm{r}}^{2}}{I^{2}}=\mathrm{I}+\frac{\pi^{2}}{\delta^{2}}\left(\frac{C_{\mathrm{r}}-C}{C}\right)^{2}
$$

and from (7) we have finally

$$
\text { (8) } \delta_{2}=\pi \frac{C_{\mathrm{r}}-C}{C} \sqrt{\frac{I^{2}}{I_{\mathrm{r}}^{2}-I^{2}}}
$$

For the case where damped oscillations exist in both primary and secondary circuits, Bjerknes ${ }^{1}$ has shown that the formula (8) holds good for the sum of both decrements $\delta_{1}$, and $\delta_{2}, \delta_{1}$ being the decrement of the primary circuit and $\delta_{2}$ that of the secondary circuit, or,

$$
\delta_{1}+\delta_{2}=\pi \frac{C_{\mathrm{r}}-C}{C} \sqrt{\frac{I^{2}}{I_{\mathrm{r}}^{2}-I^{2}}}
$$

The conditions under which this formula may be applied with sufficient accuracy are,

I. That $\delta_{1}+\delta_{2}$ be small as compared with $2 \pi$.

2. That $\frac{C_{\mathrm{r}}-C}{C}$ be small as compared with unity. 
3. That the degree of coupling between the two circuits be small.

Let us assume that it is desired to determine the logarithmic decrement of the oscillations in the aerial circuit of a radio transmitter as shown in Fig. 5. A circuit containing inductance $\mathrm{L}$, a calibrated variable condenser $\mathrm{C}$, and a sensitive low resistance hot wire instrument $\mathrm{H}$, is very loosely coupled to the aerial or antenna circuit $\mathrm{A}$. Readings of the hot-wire instrument $\mathrm{H}$, which
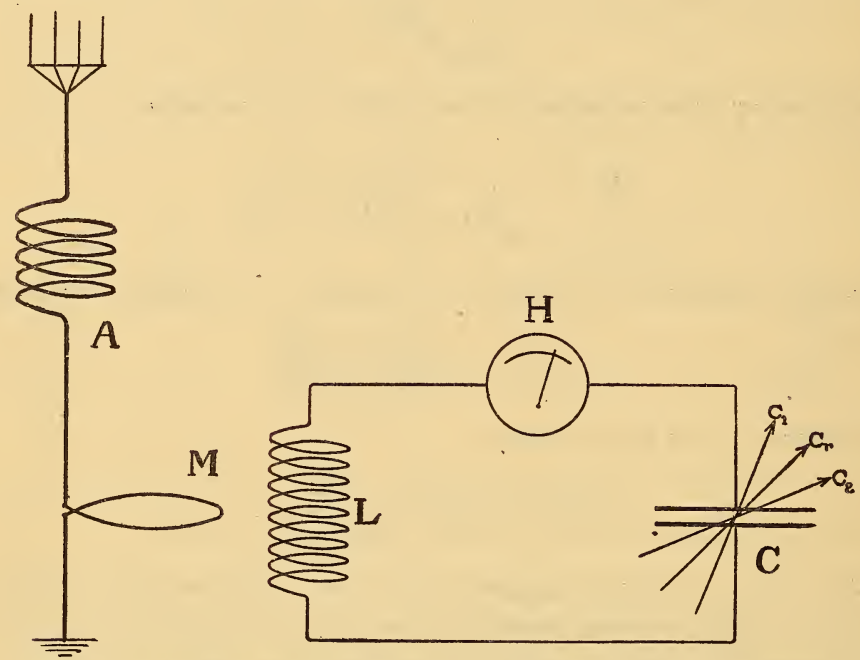

FIG. 5.-Decremeter circuit loosely coupled to the antenna circuit of a transmitter

are proportional to the square of the current in the circuit, are taken for several values of capacity $\mathrm{C}$ both sides of the resonant value $C_{\mathrm{r}}$. Plotting these readings against capacity, a resonance curve as in Fig. 6 is obtained and from one of the following formulas, ${ }^{2}$ the sum of the logarithmic decrements $\delta_{1}$ and $\delta_{2}$ may be obtained.

\footnotetext{
2 In practice it is found permissible to make the change in capacity from $C_{\mathrm{r}}$ to $C_{1}$, or from $C_{\mathrm{r}}$ to $C_{2}$ such that $I^{2}$ becomes $\frac{T}{2} I_{\mathrm{r}}{ }^{2}$, thus making the expression under the radical sign equal to unity.
} 
(9) $\delta_{1}+\delta_{2}=\pi \frac{C_{\mathrm{r}}-C_{1}}{C_{1}} \sqrt{\frac{I^{2}}{I_{\mathrm{r}}^{2}-I^{2}}}$

(I0) $\delta_{1}+\delta_{2}=\pi \frac{C_{2}-C_{\mathrm{r}}}{C_{2}} \sqrt{\frac{I^{2}}{I_{\mathrm{r}}^{2}-I^{2}}}$

(II) $\delta_{1}+\delta_{2}=\pi \frac{C_{2}-C_{1}}{C_{2}+C_{1}} \sqrt{\frac{I^{2}}{I_{\mathrm{r}}^{2}-I^{2}}}$

If the decrement $\delta_{2}$ of the measuring circuit has been previously determined, the decrement $\delta_{1}$ of the aerial circuit under test is at once obtained.

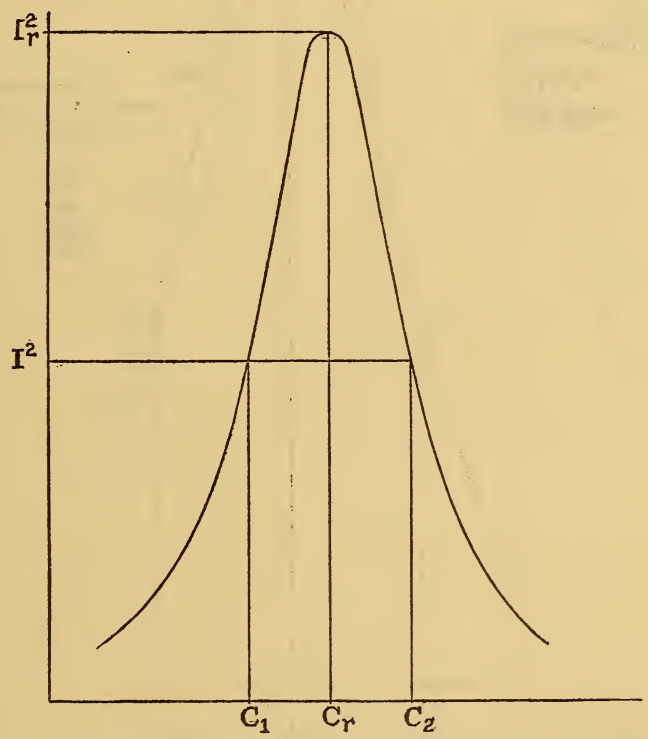

FIG. 6.--Resonance curve showing rise of current or energy in an oscillatory circuit when tuned to resonance with source of excitation

Although the measurement of the logarithmic decrement as outlined above appears to be comparatively simple, nevertheless, to obtain reasonably consistent and accurate results, the observations must be taken with considerable care, the resonance curve 
must be plotted on a large scale, and calculations must be made from several points on the curve. In fact, it is only with laboratory conveniences that satisfactory measurements can be obtained.

The instrument, which it is the purpose of this paper to describe, was designed for the purpose of facilitating the work involved in making measurements of decrement and yet permitting as great

45

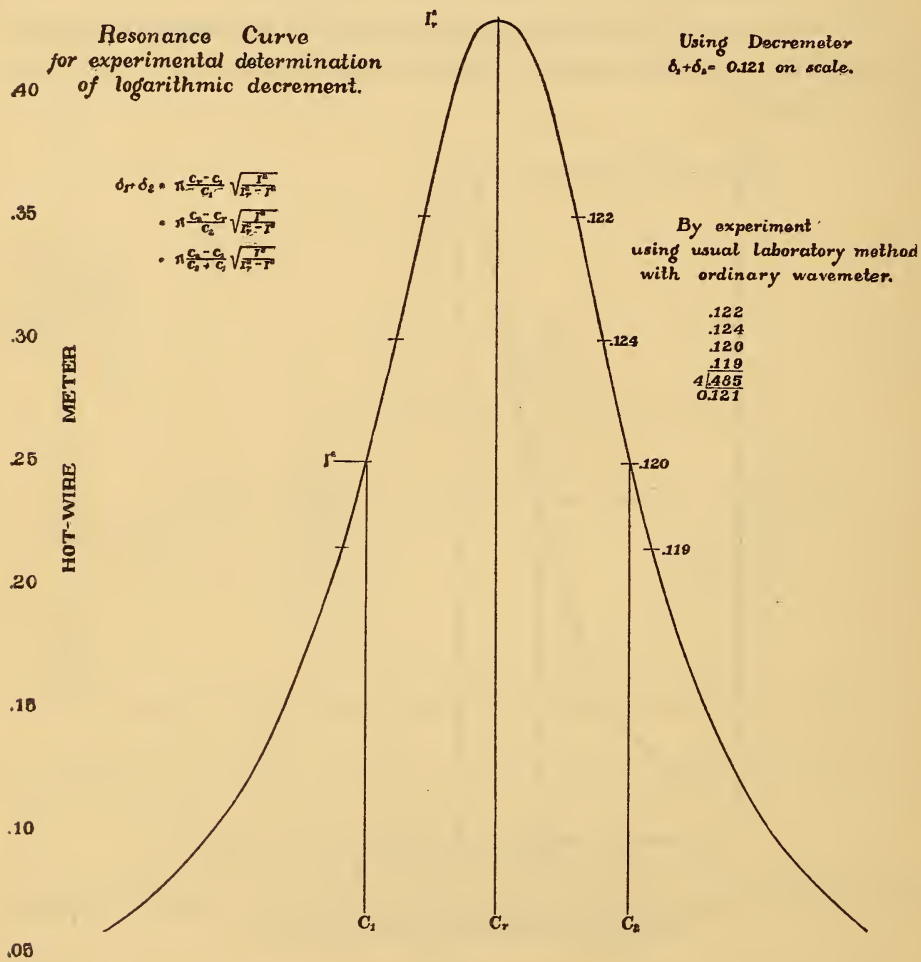

DEGREES ON CONDENSER

$\begin{array}{llllllllllllllll}93 & 94 & 95 & 96 & 97 & 98 & 99 & 100 & 101 & 102 & 103 & 104 & 105 & 106 & 107 & 108\end{array}$

FIG. 7.-Resonance curve for experimental determination of logarithmic decrement

accuracy as can be expected in the ordinary laboratory method. These requirements are particularly desirable for the purposes of 
the inspection service of the Bureau of Navigation in the enforcement of the radio communication laws. The inspection of a radio

\section{$\theta$}

8

for experimental determination of logarithmic decrement

7

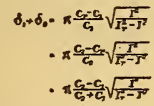

$\theta$

3

5

${ }_{5}^{8}$

1

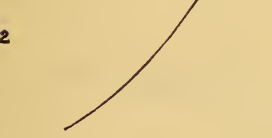

- 97

98

89

100

101

102

103

104

FIG. 8.-Resonance curve for experimental determination of logarithmic decrement

The above curve is plotted to a different scale from curve 7 : if it were plotted to the same scale it would be twice as high and only two-fifths as broad as it is, in which case it would show better the sharper resonance.

station on board ship, for example, has to be done quickly and in many cases under very unfavorable circumstances. 
In Figs. 7 and 8 typical resonance curves are shown and the task of obtaining the logarithmic decrement by the ordinary method is indicated. Identical results are obtained in a very much shorter time by means of the direct reading instrument.

\section{THEORY OF THE INSTRUMENT.}

The shape of the moving plates or surfaces of the ordinary variable condenser in common use is such that for equal angular displacements of these surfaces from the position of minimum capacity to that of maximum capacity, an approximately straight

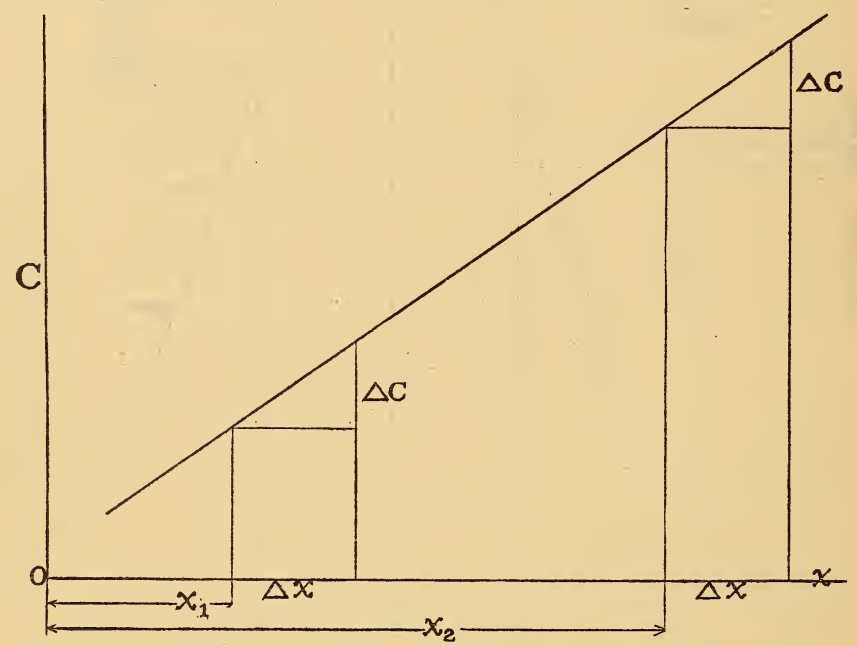

FIG. 9.-Straight line variation of capacity of the ordinary types of rotary condenser

line variation of capacity is obtained as in Fig 9. It is evident, therefore, that for any given displacement $\Delta X$ at $X=X_{1}$ the percentage change $\frac{\Delta C}{C}$ will not be equal to that obtained for an equal displacement $\Delta X$ at any other point $X=X_{2}$.

In order to make it possible to attach to a variable condenser a single predetermined scale giving values of logarithmic decrement 
corresponding to various percentage changes of capacity throughout the range of capacity of the condenser as defined by the Bjerknes formula,

$$
\delta_{1}+\delta_{2}=\pi \frac{C_{\mathrm{r}}-C}{C}, \text { for } I^{2}=\frac{1}{2} I_{\mathrm{r}}^{2}
$$

the capacity variation with equal displacements of the moving plates must be such that for any given displacement $\Delta X$ taken at any point from $X$ minimum to $X$ maximum,

$$
\frac{C_{\mathrm{r}}-C}{C}=\frac{\Delta C}{C}=\text { a constant. }
$$

The problem, therefore; of constructing a direct reading instrument for decrement measurements is largely that of determining the proper shape for the plates or surfaces of the condenser to produce a variable capacity such that for any given displacement the value of $\frac{\Delta C}{C}$ will be constant throughout the range of motion of the moving surfaces.

In other words, for a displacement of $\Delta X$, Fig. Io,

but if,

$$
\frac{C_{2}-C_{1}}{C_{1}}=\frac{C_{3}-C_{2}}{C_{2}}=\frac{C_{4}-C_{3}}{C_{n}}=\cdots \cdots \frac{C_{\mathrm{n}}-C_{\mathrm{n}-\mathrm{r}}}{C_{\mathrm{n}-\mathrm{r}}}
$$

$$
\frac{C_{2}-C_{1}}{C_{1}}=\frac{C_{3}-C_{2}}{C_{2}}
$$

then

similarly,

$$
C_{2}{ }^{2}=C_{1} C_{3} \text {, or } C_{2}=\sqrt{C_{1} C_{3}}
$$

$$
C_{3}=\sqrt{C_{2} C_{4}}
$$

or,

$$
C_{\mathrm{n}}=\sqrt{C_{\mathrm{n}-\mathrm{r}} C_{\mathrm{n}+\mathrm{x}}}
$$

It is seen, therefore, that the capacity of the variable condenser must vary in accordance with the law of geometric progression and it is now easy to formulate the equation giving the connection between the value of capacity and the position of the moving plates, 
for since the curve of capacity must obey the law of geometric progression, we have the following, Fig. I I :

$$
\text { at } x=o \text { let } C_{0}=a K^{0}=a
$$

$$
\begin{array}{rlrl}
\text { then at } x=\mathrm{I} & C_{1}=a K^{1} \\
x=2 & C_{2}=a K^{2} \\
x=3 & C_{3}=a K^{3} \\
\vdots \quad \vdots & \vdots & \vdots \\
x & =n^{\mathrm{n}} & C_{\mathrm{n}}=a K^{\mathrm{n}},
\end{array}
$$

or, in general,

$$
C=a K^{\mathrm{x}}
$$

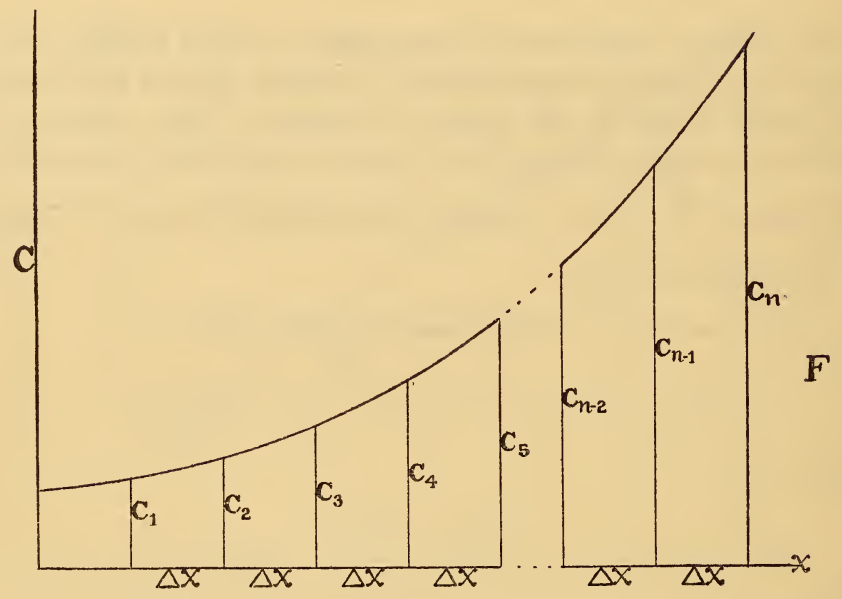

FIG. I0.-Capacity varying in accordance with the law of geometric progression

This law might have been deduced more directly as follows: The fundamental requirement of the condenser may be written:

$$
\begin{aligned}
\frac{d C}{C} & =n d x \\
\therefore \log C & =n x+h
\end{aligned}
$$

and

$$
C=\epsilon^{\mathrm{nx}+\mathrm{h}}=a \epsilon^{\mathrm{nx}}
$$


This is equivalent to equation (12). For the case of a rotary condenser where $\theta$ is the displacement angle in degrees

$$
C=a \epsilon^{\mathbf{m} \theta}
$$

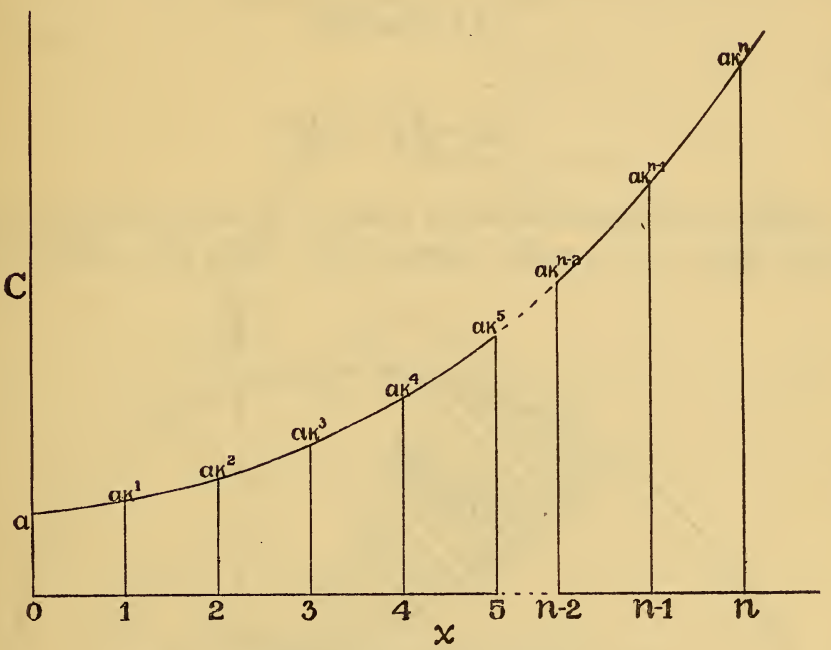

FIG. Ir.-Geometric progression

\section{DESIGN OF CONDENSER}

Since the capacity of a condenser is directly proportional to the active area of the movable surface, neglecting edge effects we may write,

$$
A=b \epsilon^{\mathrm{m} \theta}
$$

A being the area of the active surface of the moving plate, and $\theta$ the angle of displacement.

If we now consider Fig. 12 , the actual shape of the moving plate can be determined. 
By analogy with equation $\mathbf{3}$,

$$
\frac{d A}{A}=m d \theta
$$

or,

$$
d A=b m \epsilon^{\mathbf{m} \theta} d \theta
$$

but,

$$
d A=\frac{1}{2}\left(\rho^{2}-\mathrm{r}^{2}\right) d \theta
$$

$\rho$ being the distance from the center $O$ to the enveloping curve of the plate, or the radius vector, and $r$ being the radius of the

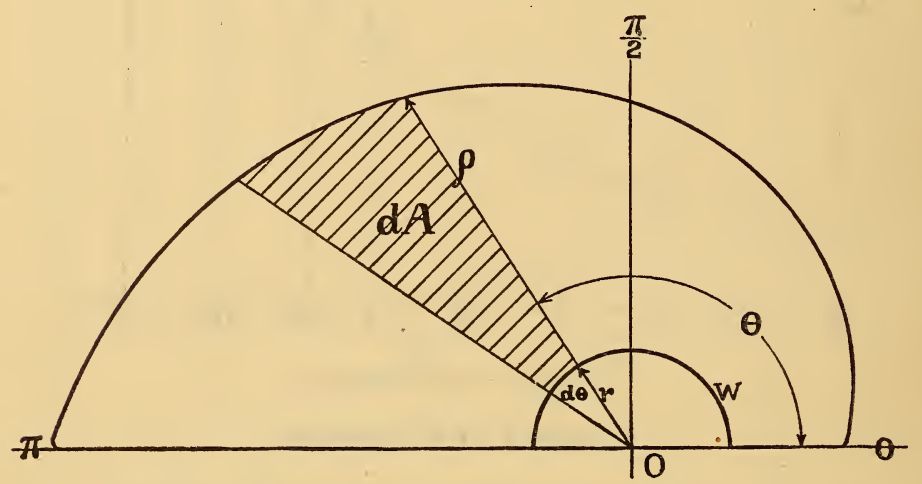

Fig. I2.- Shape of rotary plate of condenser

small circular space occupied by the separating washers between the plates.

Then

$$
\frac{\mathrm{I}}{2} \rho^{2}-\frac{\mathrm{I}}{2} \mathrm{r}^{2}=b m \epsilon^{\mathrm{m} \theta}
$$

and

$$
\rho=\sqrt{2 b m \epsilon^{\mathrm{m} \theta}+\mathrm{r}^{2}}
$$

$b$ and $m$ are constants which determine the minimum and maximum values of capacity of the condenser to be used, and having chosen these constants to suit our particular requirements, we 
can immediately determine the size and shape of our plates and construct a condenser, the capacity of which will vary in accordance with the law of geometric progression.

In Fig. I 3 are shown the stationary and rotary plates of the condenser. The stationary plates are made semicircular for convenience.

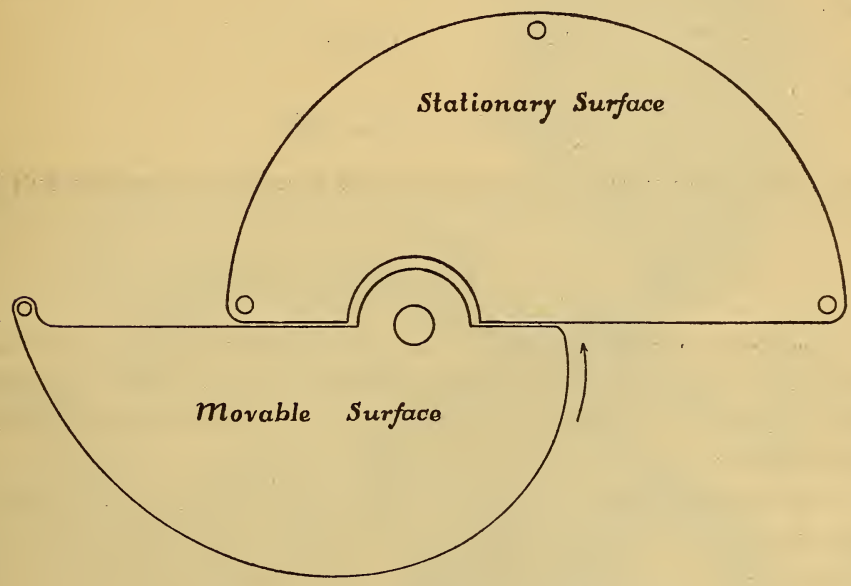

FIG. 13.-Stationary surface; movable surface

Equations 12 and 14 are identical and we may write

$$
K^{x}=\epsilon^{\mathrm{m} \theta}
$$

or

therefore

$$
x \log K=m \theta
$$

$$
m=\frac{x \log K}{\theta}
$$

If we assume that when

$$
x=\mathrm{I}, \theta=\mathrm{I} 80^{\circ}
$$

then

$$
x=\frac{\theta}{\mathrm{I} 8 \mathrm{o}}
$$


and

therefore,

$$
m=\frac{\log K}{\mathrm{I} 8 \mathrm{o}}
$$

$$
C=a \epsilon \frac{\log K}{\mathrm{I} 8 \mathrm{o}} \theta
$$

for

$$
\theta=0 \quad C_{0}=a
$$

for

$$
\theta=\mathrm{I} 80^{\circ} \quad C_{180}=a K
$$

hence, the ratio between maximum and minimum capacity will be

$$
\frac{C_{180}}{C_{0}}=K
$$

In order to obtain the ratio $K$ which has been chosen to suit our particular requirements, a fixed condenser is connected in parallel with the rotary condenser. The capacity of this fixed condenser is determined experimentally.

A rotary condenser constructed in accordance with the theory just given, with a fixed capacity connected in parallel with it, so chosen as to give the desired ratio between the maximum and minimum capacity of the combined condensers, will give a calibration curve in exact agreement with theoretical values as shown by Fig. I 4 .

\section{DETERMINATION OF DECREMENT SCALE}

It has been shown that since the capacity of the condenser to be used in the instrument varies in accordance with the law of geometric progression, the term $\frac{C_{\mathrm{r}}-C}{C}$ in the formula,

when

$$
\delta_{1}+\delta_{2}=\pi \frac{C_{\mathrm{r}}-C}{C} \sqrt{\frac{I^{2}}{I_{\mathrm{r}}^{2}-I^{2}}}=\pi \frac{C_{\mathrm{r}}-C}{C}
$$

$$
I^{2}=\frac{\mathrm{I}}{2} I_{\mathrm{r}}^{2}
$$




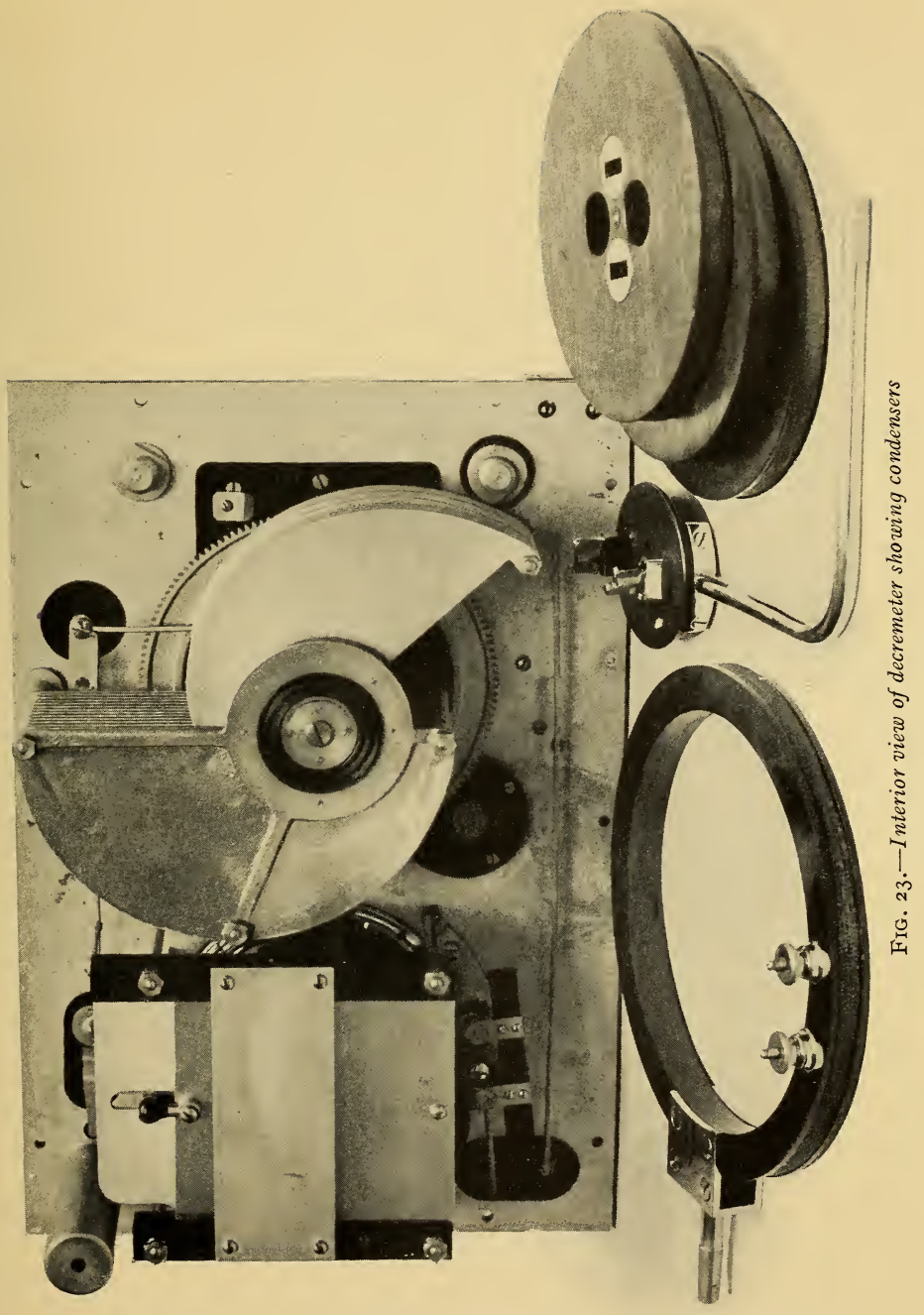


will remain constant for any given angular displacement of the rotary plates throughout the range of motion from $0^{\circ}$ to $180^{\circ}$.

In order, therefore, to predetermine a scale which can be attached to the rotary condenser and which will indicate directly

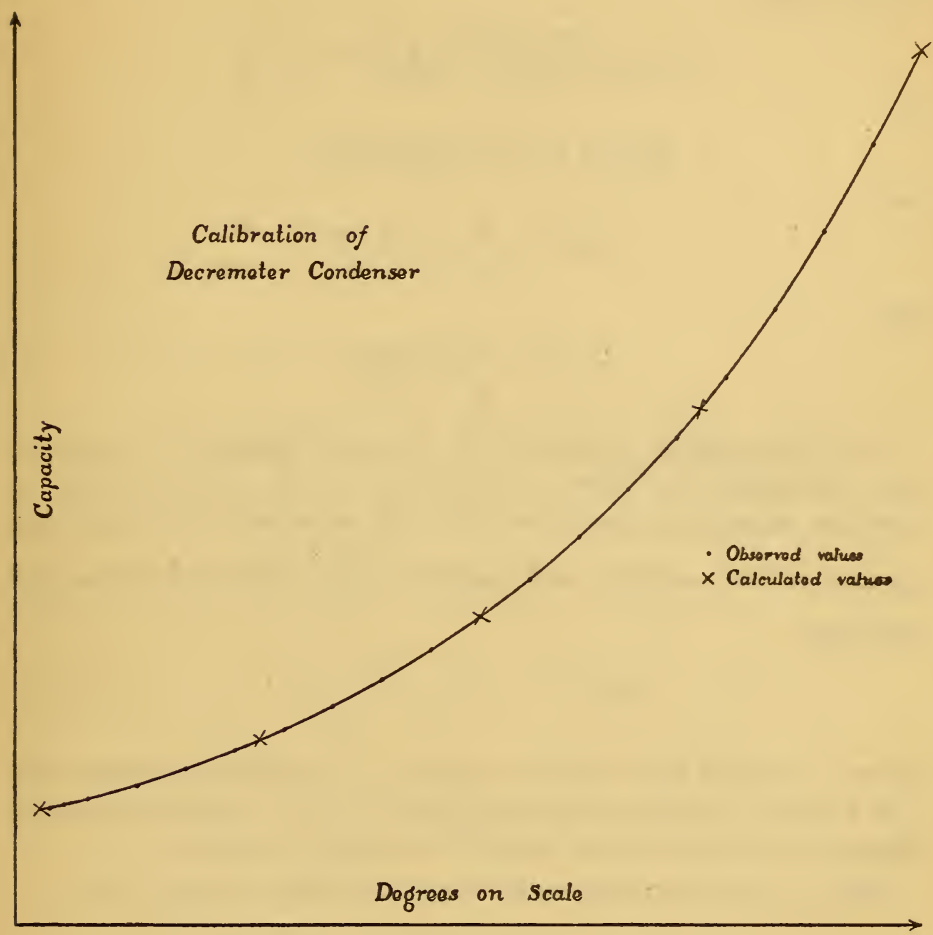

Fig. I4.-Calibration of decremeter condenser.

the value of $\delta_{1}+\delta_{2}$ for various displacements of the rotary plates, the following calculations are made.

$$
\text { Case I: } \delta_{1}+\delta_{2}=\pi \frac{C_{\mathrm{r}}-C_{1}}{C_{1}}
$$


where $C_{\mathrm{r}}$ is the value of capacity at resonance and $C_{1}$ is a smaller capacity of such a value that the current squared is reduced to one-half of its value at complete resonance.

Since $C_{\mathrm{r}}$ is proportional to $\epsilon^{\mathrm{m} \theta_{\mathrm{r}}}$ and $C_{1}$ is proportional to $\epsilon^{\mathrm{m} \theta_{1}}$ we may write

$$
\delta_{1}+\delta_{2}=\pi \frac{\epsilon^{\mathrm{m} \theta_{x}}-\epsilon^{\mathrm{m} \theta_{1}}}{\epsilon^{\mathrm{m} \theta_{1}}}=\pi\left(\epsilon^{\mathrm{m}\left(\theta_{\mathrm{r}}-\theta_{1}\right)}-\mathrm{I}\right)
$$

let

$$
\delta=\delta_{1}+\delta_{2} \text { for convenience, }
$$

then,

$$
\epsilon^{\mathrm{m}\left(\theta_{\mathrm{x}}-\theta_{1}\right)}=\frac{\delta}{\pi}+\mathrm{I}=\frac{\delta+\pi}{\pi}
$$

and

$$
\theta_{\mathrm{r}}-\theta_{1}=\frac{\mathrm{I}}{m} \log \frac{\delta+\pi}{\pi}
$$

The displacement angle $\Delta \theta=\theta_{\mathrm{r}}-\theta_{1}$ may therefore be immediately calculated for various values of $\delta=\delta_{1}+\delta_{2} . \quad m$ is as before a constant dependent upon the ratio of maximum to minimum capacity of the condenser and is equal to $\frac{\log K}{180}$, where $K$ represents this ratio.

$$
\text { Case II: } \delta_{1}+\delta_{2}=\pi \frac{C_{2}-C_{\mathrm{r}}}{C_{2}}
$$

where $C_{\mathrm{r}}$ is again the value of capacity at complete resonance and $C_{2}$ is a larger capacity of such a value that the current squared is reduced to one-half of the value at complete resonance.

Since $C_{2}$ is proportional to $\epsilon^{\mathrm{m} \theta_{2}}$ and $C_{\mathrm{r}}$ is proportional to $\epsilon^{\mathrm{m} \theta_{\mathrm{r}}}$

$$
\delta=\delta_{1}+\delta_{2}=\pi \frac{\epsilon^{\mathrm{m} \theta_{2}}-\epsilon^{\mathrm{m} \theta_{\mathrm{r}}}}{\epsilon^{\mathrm{m} \theta_{2}}}=\pi\left(\mathrm{I}-\frac{\epsilon^{\mathrm{m} \theta_{\mathrm{r}}}}{\epsilon^{\mathrm{m} \theta_{2}}}\right)
$$

then

$$
\epsilon^{\mathrm{m}\left(\theta_{2}-\theta_{\mathrm{s}}\right)}=\frac{\pi}{\pi-\delta}
$$

and

$$
\theta_{2}-\theta_{\mathrm{r}}=\frac{\mathrm{I}}{m} \log \frac{\pi}{\pi-\delta}
$$




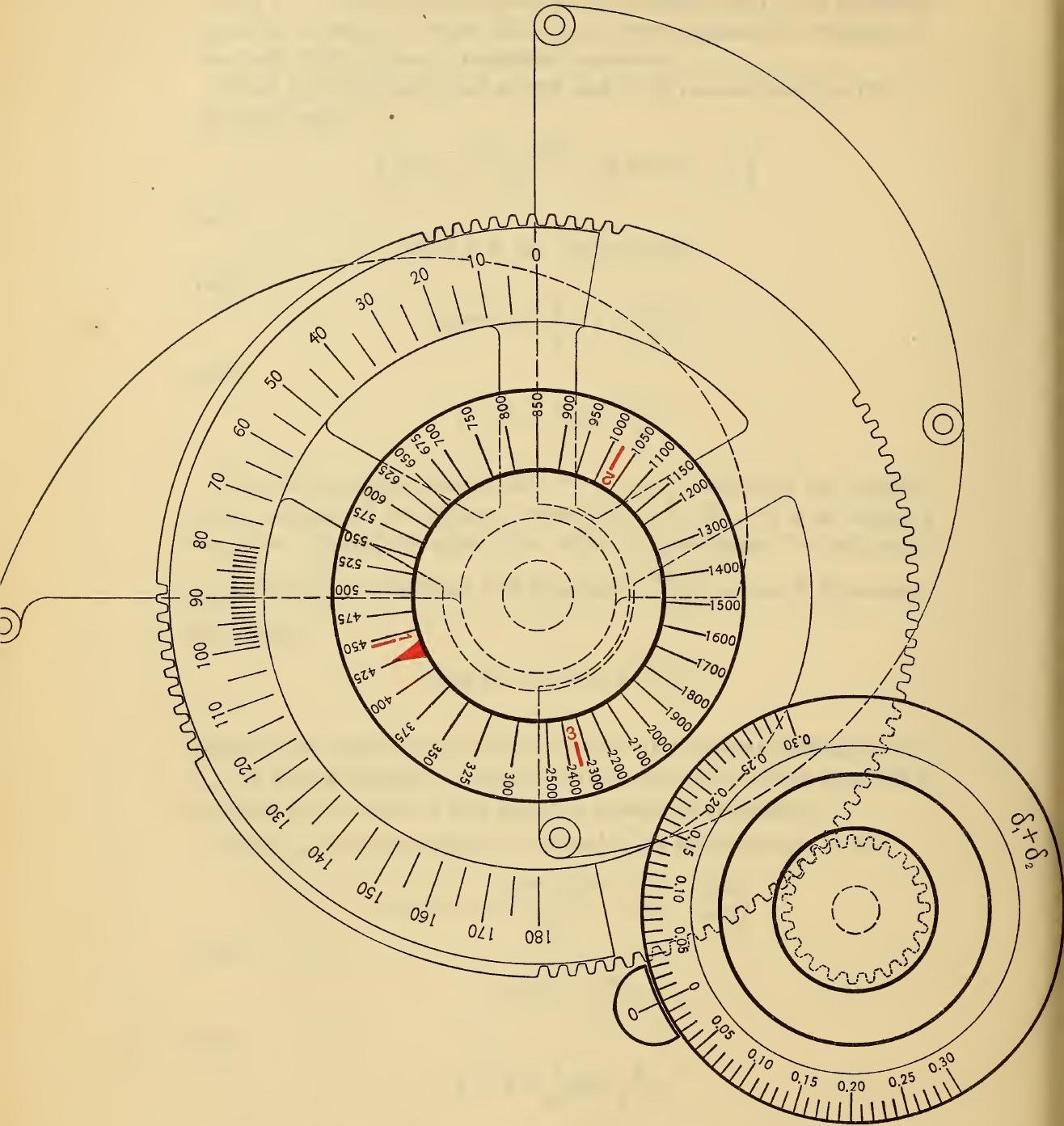

FIG. I 5.-Variable condenser, showing gears and scales mechanically attached 
and again, the displacement angle $\Delta \theta=\theta_{2}-\theta_{r}$ may be readily calculated for various values of $\delta=\delta_{1}+\delta_{2}$.

For the particular case in question, the following angles and corresponding decrements as calculated are tabulated:

\begin{tabular}{|c|c|c|}
\hline$\hat{o}_{1}+\hat{o}_{2}$ & $\begin{array}{c}\text { Case I. Reducing } \\
\text { capacity from res- } \\
\text { onance: } \theta_{\mathrm{r}}-\theta_{1}\end{array}$ & $\begin{array}{c}\text { Case II. Increas- } \\
\text { ing capacity from } \\
\text { resonance: } \theta_{2}-\theta_{\mathrm{r}}\end{array}$ \\
\hline 0.05 & $1^{\circ} .292$ & $1^{\circ} .313$ \\
0.10 & 2.564 & 2.650 \\
0.15 & 3.821 & 4.008 \\
0.20 & 5.055 & 5.389 \\
0.25 & 6.272 & 6.793 \\
0.30 & 7.472 & 8.222 \\
\hline
\end{tabular}

It should be emphasized that the formula

$$
\delta_{1}+\delta_{2}=\pi \frac{C_{\mathrm{r}}-C}{C} \sqrt{\frac{I^{2}}{I_{\mathrm{r}}^{2}-I^{2}}}
$$

does not strictly apply in cases where $\delta_{1}+\delta_{2}$ is great in comparison with $2 \pi$ and $\frac{C_{\mathrm{r}}-C}{C}$ is great in comparison with unity.

For $\delta_{1}+\delta_{2}=0.2$ the formula may still be applied for practical purposes with reasonable accuracy. In the foregoing tabulation calculations have been made for $\delta_{1}+\delta_{2}$ as high as 0.3 , but the method and formula should preferably not be applied at values of $\delta_{1}+\delta_{2}$ greater than 0.2 .

It will be noted that the angles tabulated above are very small, and if the decrement scale were attached directly to the shaft of the condenser it would be extremely short and difficult to read.

In order to open out the scale, it is geared to the condenser shaft at a 6-to-I ratio, as shown in Fig. I 5 .

Furthermore, the decrement readings are taken in such a way as to simultaneously include both measurements as defined by cases I and II. The displacement angle is then the sum of the angles tabulated under these two cases. 


$$
\Delta \theta=\theta_{2}-\theta_{\mathrm{r}}+\theta_{\mathrm{r}}-\theta_{1}=\theta_{2}-\theta_{1}
$$

The value of this angle $\Delta \theta=\theta_{2}-\theta_{1}$ could have been calculated directly from the formula

$$
\delta_{1}+\delta_{2}=\pi \frac{C_{2}-C_{1}}{C_{2}+C_{1}}
$$

for since $C_{2}$ is proportional to $\epsilon^{\mathrm{m} \theta_{2}}$ and $C_{1}$ is proportional to $\epsilon^{\mathrm{m} \theta_{1}}$, then carrying out the method used in cases I and II we get directly,

$$
\theta_{2}-\theta_{1}=\frac{1}{m} \log \frac{\pi+\delta}{\pi-\delta}
$$

The final graduations for the decrement scale are obtained by multiplying $\theta_{2}-\theta_{1}$ by the gear ratio of 6 , as in the following table:

\begin{tabular}{|l|c|c|}
\hline$\delta_{1}+\delta_{2}$ & $\theta_{2}-\theta_{1}$ & $\left(\theta_{2}-\theta_{1}\right) \times 6$ \\
\hline 0. & 0 & 0 \\
0.05 & $2^{\circ} .605$ & $15^{\circ} .63$ \\
0.10 & 5.214 & 31.28 \\
0.15 & 7.830 & 46.98 \\
0.20 & 10.444 & 62.70 \\
0.25 & 13.065 & 78.39 \\
0.30 & 15.694 & 94.20 \\
\hline
\end{tabular}

The decrement scale is marked to the left and to the right of zero in accordance with this table.

\section{THE MEASUREMENT OF LOGARITHMIC DECREMENT}

Considering now Fig. I6, the operation for measuring the logarithmic decrement is as follows:

The rotary condenser is first set at the position of complete resonance as indicated by the maximum deflection of the sensitive hot-wire instrument, the scale readings of which are proportional to the current squared. This maximum deflection is now reduced to one-half its value by decreasing or increasing the capacity of the rotary condenser. The decrement scale, which may be rotated independently, is now set at zero, then clamped so that when the condenser is again varied it will rotate with it.

Starting at the zero setting with the hot-wire instrument reading one-half the maximum deflection, the condenser is varied continu- 
ously in one direction until the needle of the hot-wire instrument makes a complete excursion from one-half deflection to maximum deflection and back again to one-half deflection. The scale reading now opposite the index mark $\mathrm{O}$ is the value of $\delta_{1}+\delta_{2}, \delta_{1}$ being the

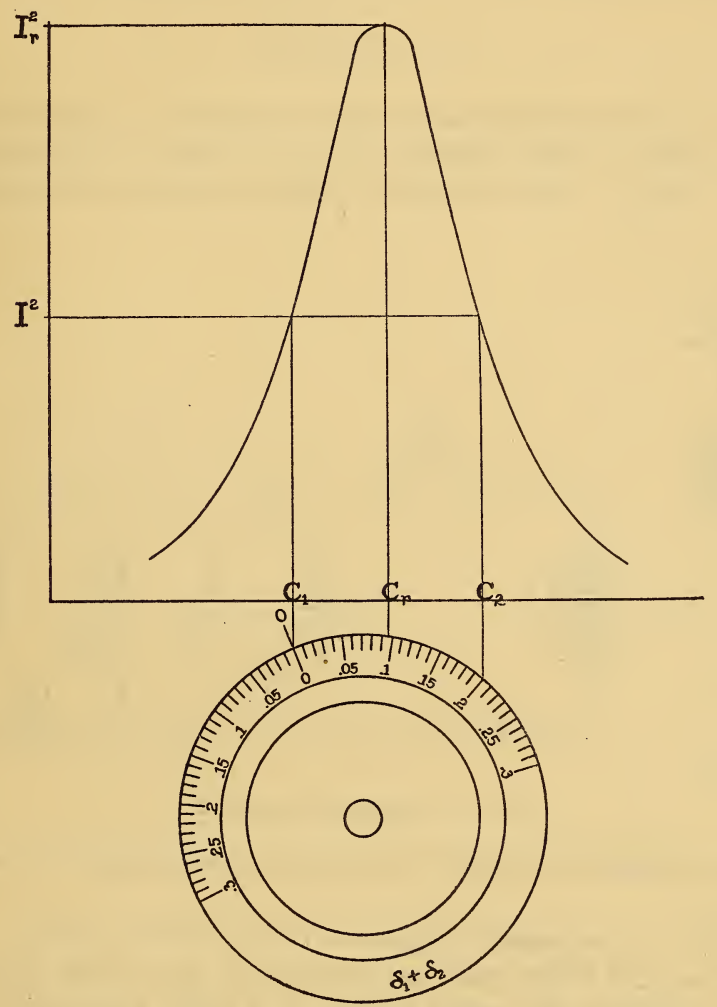

Fig. 16.-Diagram showing relation between decrement scale and resonance curve

decrement of the circuit under test and $\delta_{2}$ the known decrement of the instrument.

It will be noted by referring to Fig. I 6 that it is desirable to make the zero setting of the decrement scale at the point of half 
deflection and also to take the final reading at the point of half deflection, because at these points the resonance curve is steep, and consequently the settings are sharply defined and easily made. In this connection it will be noted that the formula

$$
\delta_{1}+\delta_{3}=\pi \frac{C_{2}-C_{1}}{C_{2}+C_{1}}
$$

does not involve the resonant value of capacity, $C_{r}$, but only those at the points of half deflection where the slope of the resonance curve is steep. This formula is therefore the most desirable one
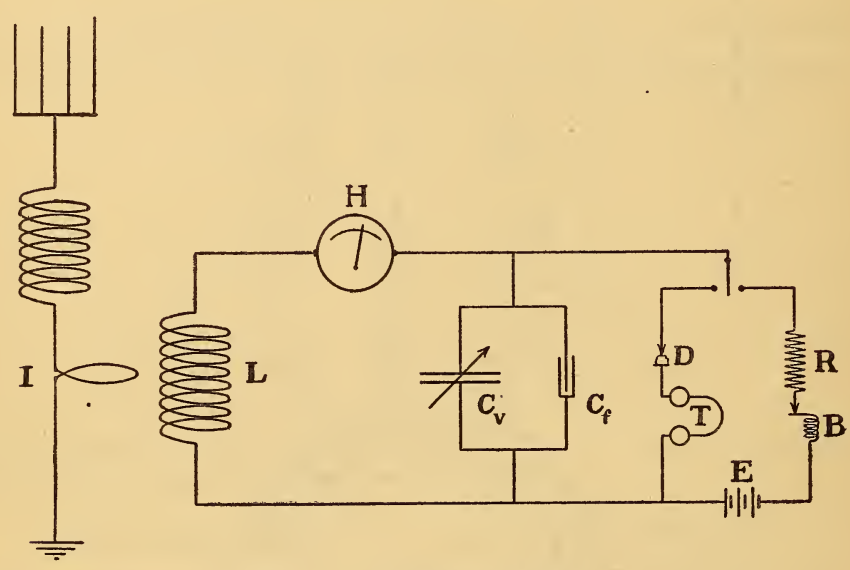

FIG. 17.-Diagram of connections

to use, and the decremeter is consequently operated in accordance with it.

In Fig. I 7 a schematic diagram of the circuit is shown. $I$ is a single-turn coil which may be connected in the circuit under test, as, for example, the aerial circuit of a radio transmitter. The inductance of this single turn is, in the majority of practical cases, small as compared with the total inductance of the circuit under test, and therefore will not affect the tuning adjustment.

The coil $\mathrm{L}$ is the inductance of the decremeter circuit and is so arranged that the mutual inductance between it and coil $I$ can be 


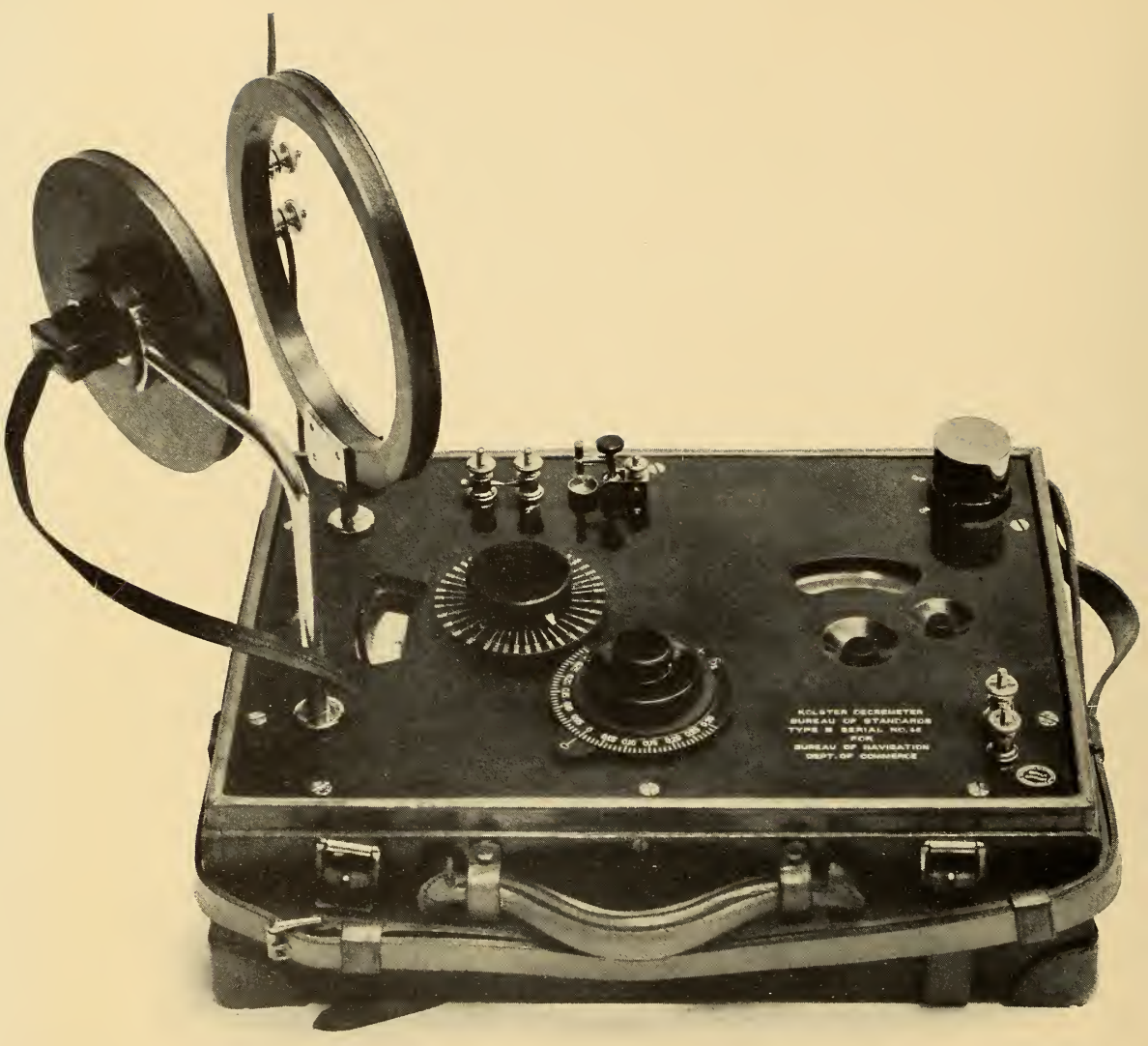

FIG. 22.-Decremeter set up for operation 
easily varied. It is very essential that the degree of coupling between the circuit under test and the decremeter circuit be small.

$C_{v}$ is the variable condenser to which the decrement scale is attached through gears. In parallel with $C_{v}$ is a small condenser $C_{f}$ which remains fixed in value after proper adjustment.

$H$ represents the hot-wire instrument or indicating device, the scale of which is so marked that the readings are proportional to the square of the current passing through it.

A crystal dector $D$ is provided and the wave length of distant stations may be measured by using telephone receivers $T$.

By means of a switch, the buzzer circuit $R B E$ may be connected to the instrument for calibration purposes.

Fig. 18 shows a top view of the instrument in detail.

\section{EXPERIMENTAL DATA}

In order to determine the accuracy of the instrument for measurement of $\delta_{1}+\delta_{2}$ the following experiments were made:

Experiment 1 . - The decremeter was used as an ordinary wave meter, loosely coupled to the secondary of a quenched spark transmitter. A resonance curve was obtained similar to those shown in Figs. 7 and 8 and for several ratios $\frac{I_{\mathrm{r}}^{2}}{I^{2}}, \delta_{1}+\delta_{2}$ was calculated from the formula

$$
\delta_{1}+\delta_{2}=2 \pi \frac{\lambda_{2}-\lambda_{1}}{\frac{\lambda_{2}+\lambda_{1}}{I_{\mathrm{r}}^{2}-I^{2}}}
$$

and the following values obtained:

\begin{tabular}{|c|c|}
\hline$\frac{\mathbf{I r}}{\mathbf{I}^{2}}$ & $\delta_{1}+\delta_{2}$ \\
\hline 1.180 & 0.0970 \\
1.475 & 0.0893 \\
1.735 & 0.0911 \\
2.000 & 0.0903 \\
\hline Average & 0.0919 \\
\hline
\end{tabular}


A single measurement obtained by means of the decremeter used as a direct reading instrument gave at once a value of 0.091 for $\delta_{1}+\delta_{2}$.

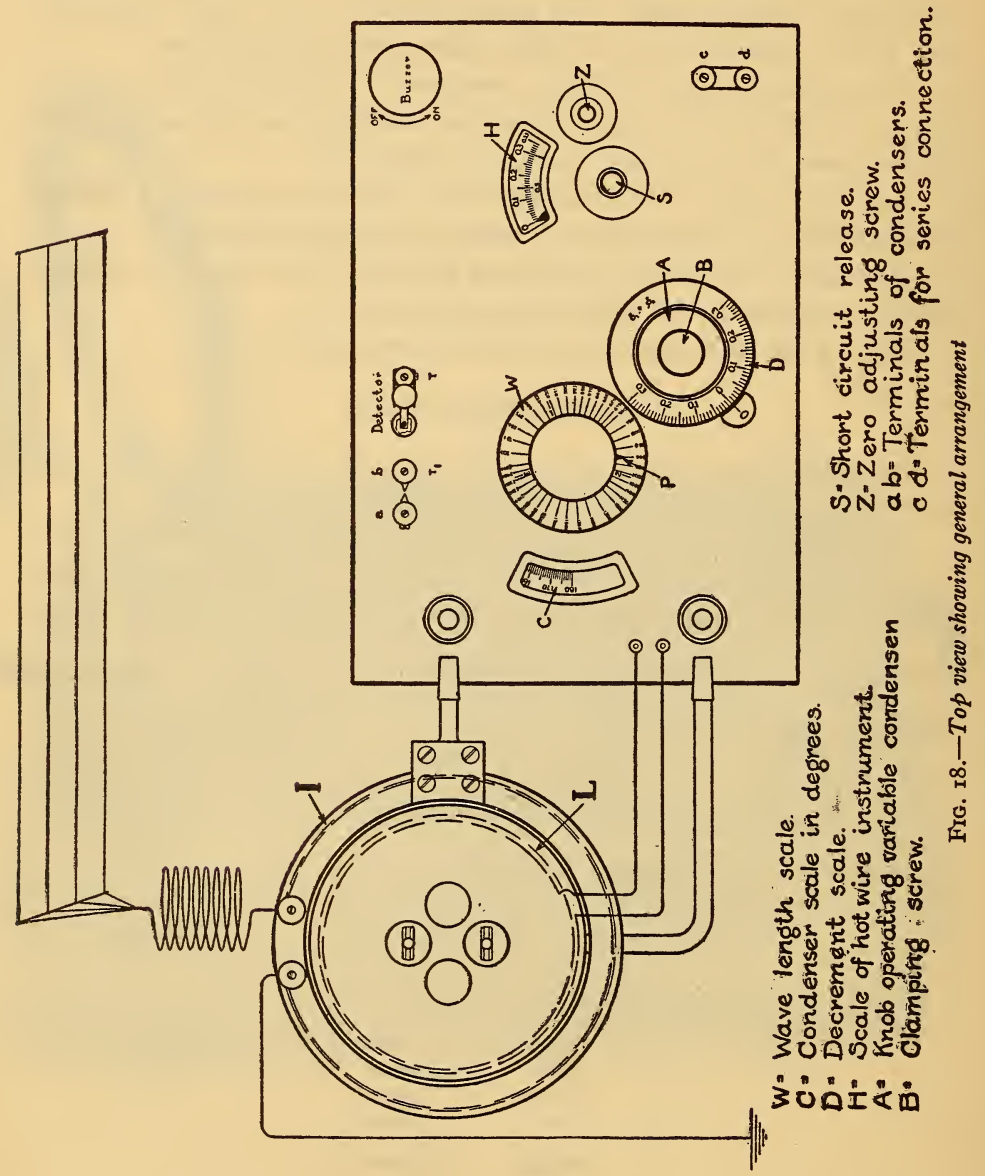

A further check was obtained by calculating the required value of $\theta_{2}-\theta_{1}$ for $\delta_{1}+\delta_{2}=0.09$ I from the formula 


$$
\begin{aligned}
& \theta_{2}-\theta_{1}=\frac{I}{m} \log \frac{\pi+\delta}{\pi-\delta} \text { for } I^{2}=\frac{I}{2} I_{\mathrm{I}}^{2} \\
& \theta_{2}-\theta_{1}=4^{\circ} .75 \text { by calculation } \\
& \theta_{2}-\theta_{1}=4^{\circ} .68 \text { as determined from the experi- }
\end{aligned}
$$
mentally obtained resonance curve.

Experiment 2.- In this experiment the decremeter was again loosely coupled to the secondary circuit of a quenched spark transmitter and a direct measurement made of $\delta_{1}+\delta_{2}$. A resistance, in the form of a short straight piece of about No. 40 manganin wire, was then inserted in the circuit of the instrument and a direct measurement made of $\delta_{1}+\delta_{2}+\Delta \delta_{2}, \Delta \delta_{2}$ being the additional decrement due to the inserted resistance.

The capacity of the condenser and the frequency of the oscillations being known, the value of the inserted resistance $R$ was calculated from the formula

$$
R=\frac{\Delta \delta_{2}}{\pi C \omega}
$$

and the following results obtained.

Test No. 1

\begin{tabular}{|r|r|}
\hline $\begin{array}{c}\hat{o}_{1}+\delta_{2} \text { read from } \\
\text { decrement scale } \\
\text { of instrument }\end{array}$ & $\begin{array}{c}\delta_{1}+\hat{\delta}_{2}+\Delta \delta_{2} \text { read } \\
\text { from decrement } \\
\text { scale of instrument }\end{array}$ \\
\hline 0.132 & 0.168 \\
0.130 & 0.169 \\
0.130 & 0.163 \\
0.131 & 0.172 \\
0.130 & 0.167 \\
$\overline{\text { Average }=0.131}$ & Average $=0.168$ \\
\hline
\end{tabular}

$$
\begin{aligned}
& \delta_{1}+\delta_{2}+\Delta \delta_{2}=0.168 \\
& \delta_{1}+\delta_{2}=0.13 \mathrm{I} \\
& \therefore \quad \Delta \delta_{2}=0.037
\end{aligned}
$$


Capacity of condenser at resonance $=334 \mu$. $\mu$. .

$$
\begin{aligned}
& \omega=2 \pi n=3.66 \times 10^{6} . \\
& R=\frac{\Delta \delta_{2}}{\pi C \omega}=9.63 \Omega
\end{aligned}
$$

By measurement on D. C. bridge,

$$
R=9.5 \mathrm{I} \Omega
$$

Another test was made at a different frequency and consequently with a different value of capacity.

Test No. 2

$$
\begin{aligned}
\delta_{1}+\delta_{2}+\Delta \delta_{2} & =0.155 \\
\delta_{1}+\delta_{2} & =0.099 \\
\Delta \delta_{2} & =0.056
\end{aligned}
$$

Capacity of condenser at resonance $=764 \mu . \mu . f$.

$$
\begin{aligned}
& \omega=2 \pi n=2.47 \times 10^{6} \\
& R=\frac{\Delta \delta_{2}}{\pi C \omega}=9.45 \Omega
\end{aligned}
$$

Value of $R$ measured on D. C. bridge $=9.5 \mathrm{I}$.

$$
\begin{array}{r}
\text { Test No. } \mathrm{I}: R=9.63 \Omega \\
\text { Test No. } 2: R=9.45 \Omega \\
\text { Average }=9.54 \Omega
\end{array}
$$

Experiment 3.- In this case resistance was inserted in the secondary circuit of the transmitter and the value of this resistance calculated in the same manner as in experiment 2 .

\section{Test 1}

$$
\begin{aligned}
\delta_{1}+\Delta \delta_{1}+\delta_{2} & =0.14 \mathrm{I} \\
\delta_{1}+\delta_{2} & =0.089 \\
\Delta \delta_{1}= & =0.052
\end{aligned}
$$


Capacity of condenser at resonance $=3900 \mu$. . . .

$$
\begin{aligned}
\omega & =2 \pi n=3.35 \times 10^{6} \\
R=\frac{\Delta \delta_{1}}{\pi C \omega} & =1.27 \Omega
\end{aligned}
$$

Value of $R$ measured on D. C. bridge $=I .242 \Omega$

Test No. 2

$$
\begin{aligned}
\delta_{1}+\Delta \delta_{1}+\delta_{2} & =0.1 \text { II } \\
\delta_{1}+\quad \delta_{2} & =0.074 \\
\Delta \delta_{1} & =0.037
\end{aligned}
$$

Capacity of condenser at resonance $=3900 \mu$. $\mu . f$.

$$
\begin{aligned}
& \omega=2.43 \times 10^{6} \\
& R=\frac{\Delta \delta_{1}}{\pi C \omega}=\mathrm{I} .24 \Omega
\end{aligned}
$$

Test No. $\mathrm{I}: R=\mathrm{I} .27 \Omega$

Test No. 2: $R=\mathrm{I} \cdot 24 \Omega$

$$
\text { Average }=1.255 \Omega
$$

\section{DETERMINATION OF WAVE-LENGTH SCALE}

Since the capacity of the variable condenser in the instrument varies according to a definitely known law, it is possible to attach to this condenser a predetermined scale indicating wave lengths directly. The graduations of the wave length scale are determined by calculation in the following manner:

It has been shown that the capacity of the condenser may be expressed as $C=a \epsilon^{\mathrm{m} \theta}$.

The wave length $\lambda$ is proportional to $\sqrt{C}$

therefore, $\quad \lambda$ is proportional to $\sqrt{\epsilon^{\mathrm{m} \theta}}$

or

$\lambda$ is proportional to $\epsilon^{\frac{m \theta}{2}}=\epsilon^{\mathrm{n} \theta}$

where

$$
n=\frac{m}{2}
$$


Now let $\lambda_{1}$ be any wave length within the range of the instrument, and $\lambda_{2}$ any other wave length desired, then

and

$$
\frac{\lambda_{2}}{\lambda_{1}}=\frac{\epsilon^{\mathrm{n} \theta_{2}}}{\epsilon^{\mathrm{n} \theta_{1}}}=\epsilon^{\mathrm{n}\left(\theta_{2}-\theta_{1}\right)}
$$

$$
\log \frac{\lambda_{2}}{\lambda_{1}}=n\left(\theta_{2}-\theta_{1}\right)
$$

or

therefore

$$
\theta_{2}-\theta_{1}=\frac{1}{n} \log \frac{\lambda_{2}}{\lambda_{1}}=\frac{2}{m} \log \frac{\lambda_{2}}{\lambda_{1}}
$$

$$
\theta_{2}=\theta_{1} \pm \frac{2}{m} \log \frac{\lambda_{2}}{\lambda_{1}}
$$

For the purpose of determining the graduations for the scale, $\lambda_{1}$ may be any wave length, as, for example, 300 meters. Furthermore, $\theta_{1}$ may be taken as zero, for convenience, then

$$
\theta_{2}= \pm \frac{2}{m} \log \frac{\lambda_{2}}{300}
$$

From this equation $\theta_{2}$ may be calculated for any wave length $\lambda_{2}$. The scale will appear as shown in Fig. I9.

The scale is arranged so that it can rotate about the shaft of the variable condenser independently but remains stationary when the condenser is rotated. A pointer is attached to the shaft of the condenser and travels over the scale as the condenser rotates.

In order to cover a wide range of wave lengths, several coils are supplied with the instrument, each coil covering a part of the range. It is necessary, therefore, to adjust the position of the wave length scale to correspond with the particular coil in circuit. The red marks shown in Fig. I9 indicate the maximum wave length obtainable with the coils $\mathrm{I}, 2$, and 3 , respectively. The position of these red marks is determined experimentally.

\section{THE MEASUREMENT OF WAVE LENGTH}

The variable condenser is first set at $180^{\circ}$, the wave length scale is then adjusted so that the red mark on the scale corresponding to the coil in circuit is directly under the pointer attached to the 


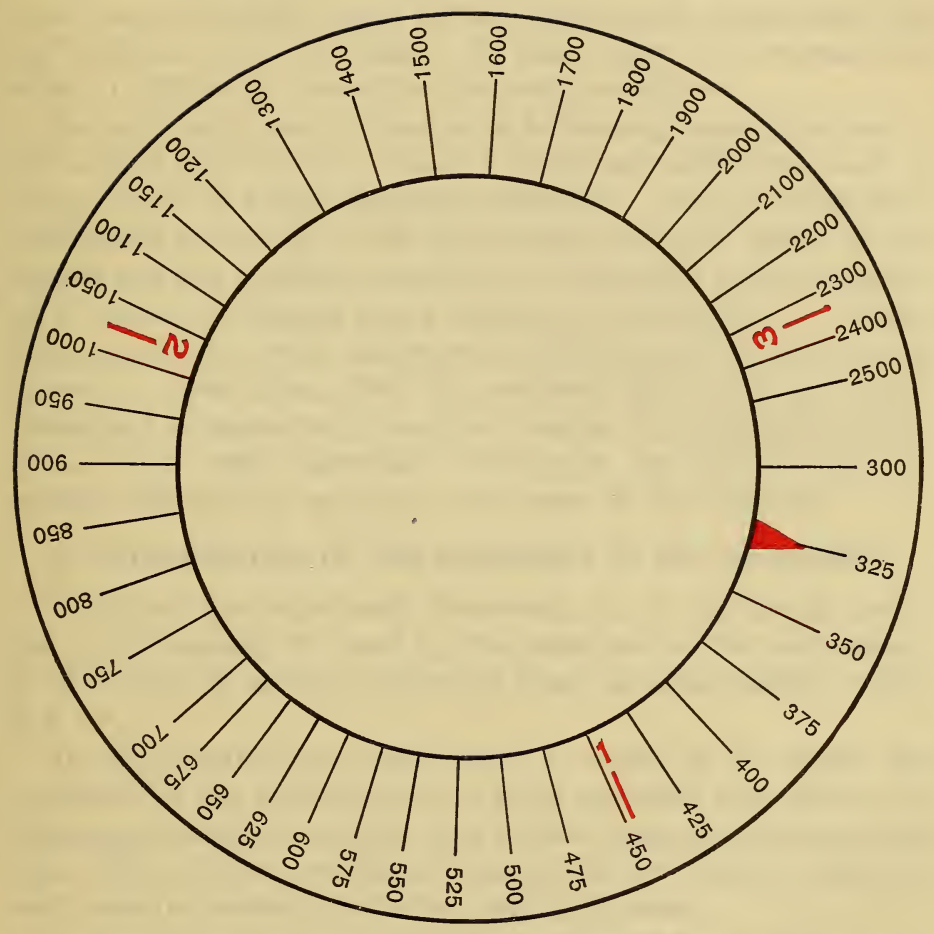

FIG. I9.-Wave-length scale 
condenser shaft. The wave length scale remains in this position and, as the condenser is varied, the pointer travels over the scale, indicating the wave length when resonance is obtained, as shown by the maximum reading of the hot wire instrument needle.

When the instrument is used as a receiver with detector and telephones, or as a transmitter using the buzzer, the wave length scale does not strictly apply for the wave length range below the $90^{\circ}$ position of the condenser. In these cases it is necessary to refer to calibration curves for the small correction.

The instrument may be used in an interesting manner for receiving audible signals from a source of undamped oscillations, such as an arc circuit or a high frequency alternator. To accomplish this, oscillations are set up in the wave meter circuit by means of the buzzer and the telephone receivers are connected to the detector at $T$ and to the binding post $a$ instead of $b$, see figure 18 . Under these conditions, when undamped oscillations are induced in the circuit, a heterodyne effect is produced and the wave meter becomes a comparatively sensitive receiver of undamped oscillations. Very weak harmonics existing in arc circuits may be readily measured by using the instrument in this manner.

\section{DETERMINATION OF THE DECREMENT OF THE INSTRUMENT}

To obtain the logarithmic decrement, $\delta_{1}$, of the circuit under test, it is necessary to know $\delta_{2}$, the decrement of the instrument, in order that it may be subtracted from the scale reading which is $\delta_{1}+\delta_{2}$.

An ideal method for determining $\delta_{2}$ would be to charge the condenser of the instrument at a given potential and allow it to discharge through the circuit, first without inserted resistance and then with a known resistance inserted in the circuit, noting in each case the reading of the hot wire instrument.

The energy in the circuit in both cases would then be equal and,

$$
I_{1}^{2} R=I_{2}^{2}(R+\Delta R)
$$

where $R$ is the resistance of the circuit and $\Delta R$ is the resistance inserted. Then,

$$
\dot{R}=\Delta R \frac{I_{2}^{2}}{\bar{I}_{1}^{2}-I_{2}^{2}}
$$


where $I_{2}{ }^{2}$ and $I_{1}{ }^{2}$ represent, respectively, the readings of the hot wire instrument with and without inserted resistance, these readings being proportional to the square of the current flowing in the circuit.

If the inductance $L$ or capacity $C$ of the circuit are known, $\delta_{2}$ is then determined for any value of $\omega$, since,

$$
\delta_{2}=\pi R C \omega=\pi \frac{R}{L \omega}
$$

A method used in practice which approaches this ideal case very closely is as follows:

Energy is supplied to the instrument by means of impact excitation, in which case nearly free oscillations exist in the circuit of the instrument. These oscillations, therefore, have a frequency and damping determined by the constants of the circuit. To determine the resistance of the circuit, readings of the hot wire instrument are taken with and without inserted resistance. The energy in the circuit, however, would not in practice be strictly equal in the two cases and

or

$$
\begin{gathered}
I_{1}^{2} R=K I_{2}^{2}(R+\Delta R) \\
R=\Delta R \frac{K I_{2}{ }^{2}}{I_{1}{ }^{2}-K I_{2}{ }^{2}}
\end{gathered}
$$

It has been shown in previous works ${ }^{3}$ on this subject that

$$
K=\mathrm{I}+\frac{\Delta \delta}{\delta_{1}+\delta_{2}}
$$

Where $\delta_{1}$ is the decrement of the exciting circuit, $\delta_{2}$ the decrement of the instrument circuit, and $\Delta \delta$ the additional decrement due to the insertion of a small resistance $\Delta R$.

It is seen that for the case of impact excitation where $\delta_{1}$ is very large as compared to $\Delta \delta, K$ will be very nearly unity and for practical purposes we may write

$$
R=\Delta R \frac{I_{2}{ }^{2}}{I_{1}{ }^{2}-I_{2}{ }^{2}}=\Delta R \frac{\mathrm{I}}{\frac{I_{1}{ }^{2}}{I_{2}{ }^{2}}-\mathrm{I}}
$$


Where it is desired to make $\Delta R$, the inserted resistance equal to $R$, the resistance of the instrument circuit, which corresponds to making $\Delta \delta$ equal to $\delta_{2}$, then for the case of impact excitation,

$$
\frac{I_{1}^{2}}{I_{2}^{2}}=2
$$

On the other hand, if $\delta_{1}=0$ as in the case of undamped oscillations,

$$
K=2 \text { and } \frac{I_{1}{ }^{2}}{I_{2}^{2}}=4
$$

In general, therefore, when it is desired to make the inserted resistance $\Delta R$ equal to the resistance of the instrument $R$, the amount by which $I_{1}{ }^{2}$ must be reduced or the ratio of $\frac{I_{1}{ }^{2}}{I_{2}{ }^{2}}$ depends upon the ratio of $\delta_{1}$ to $\delta_{2}$ for when $\Delta \delta=\delta_{2}$

and for

$$
K=\mathrm{I}+\frac{\delta_{2}}{\delta_{1}+\delta_{2}}=\mathrm{I}+\frac{\mathrm{I}}{\frac{\delta_{1}}{\delta_{2}}+\mathrm{I}}
$$

$$
\begin{aligned}
& \delta_{1}=0 \quad K=2 \text { and } \frac{I_{1}{ }^{2}}{I_{2}{ }^{2}}=4 \\
& \delta_{1}=\infty K=\mathrm{I} \text { and } \frac{I_{1}{ }^{2}}{I_{2}{ }^{2}}=2
\end{aligned}
$$

For intermediate values of $\frac{\delta_{1}}{\delta_{2}}$ between o and $\infty, K$ will vary from 2 to $\mathrm{I}$ and the ratio $\frac{I_{1}{ }^{2}}{I_{2}{ }^{2}}$ correspondingly from 4 to 2 .

The most direct and simple method, however, for obtaining $\delta_{2}$ is to excite the instrument by means of undamped oscillations, then

$$
\delta_{2}=\pi \frac{C_{2}-C_{1}}{C_{2}+C_{1}} \sqrt{\frac{I^{2}}{I_{\mathrm{r}}^{2}-I^{2}}}
$$

as shown in the earlier part of this paper. 
If suitable means for producing undamped oscillation are not available the method of impact excitation is very satisfactory, provided that $\delta_{1}$ is very large as compared with $\Delta \delta$.

The curves in Fig. 20 give the values of $\delta_{2}$ for coils I, 2, and 3 ,

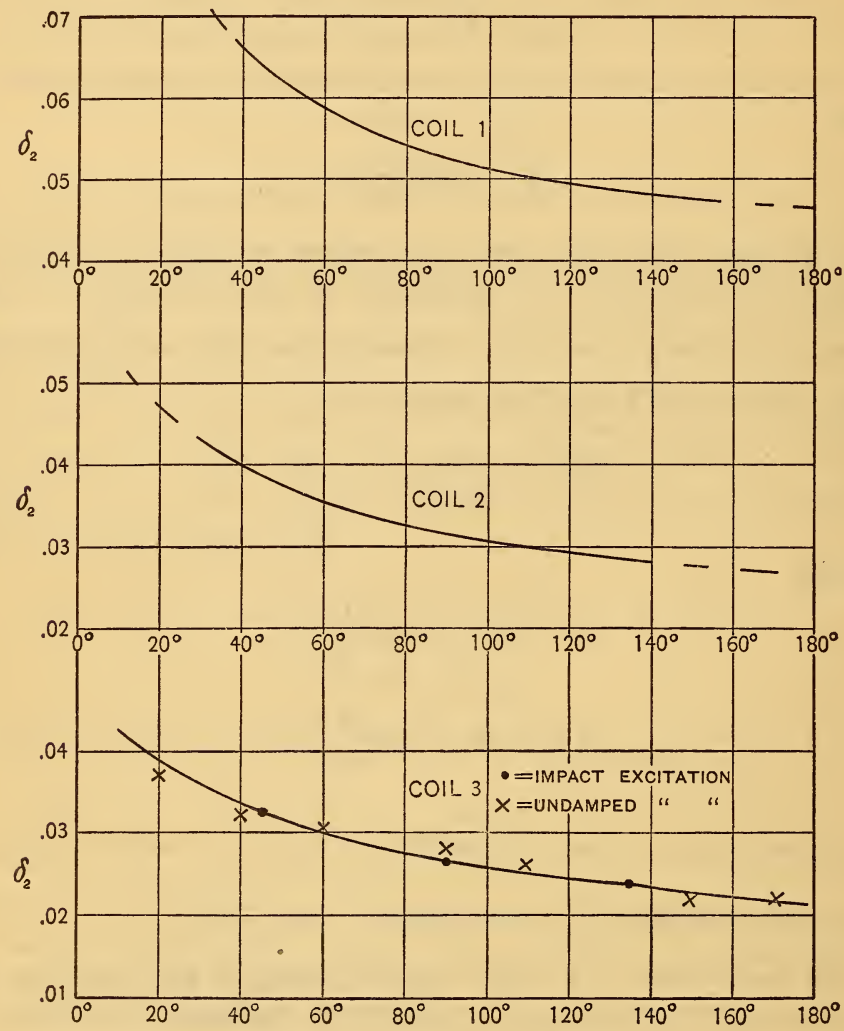

FIG. 20.-Decrement of instrument

at various settings of the variable condenser. These values were obtained by the method of impact excitation.

On curve 3 are shown values of $\delta_{2}$ obtained by using undamped oscillations from a Poulsen arc as a source of excitation. 


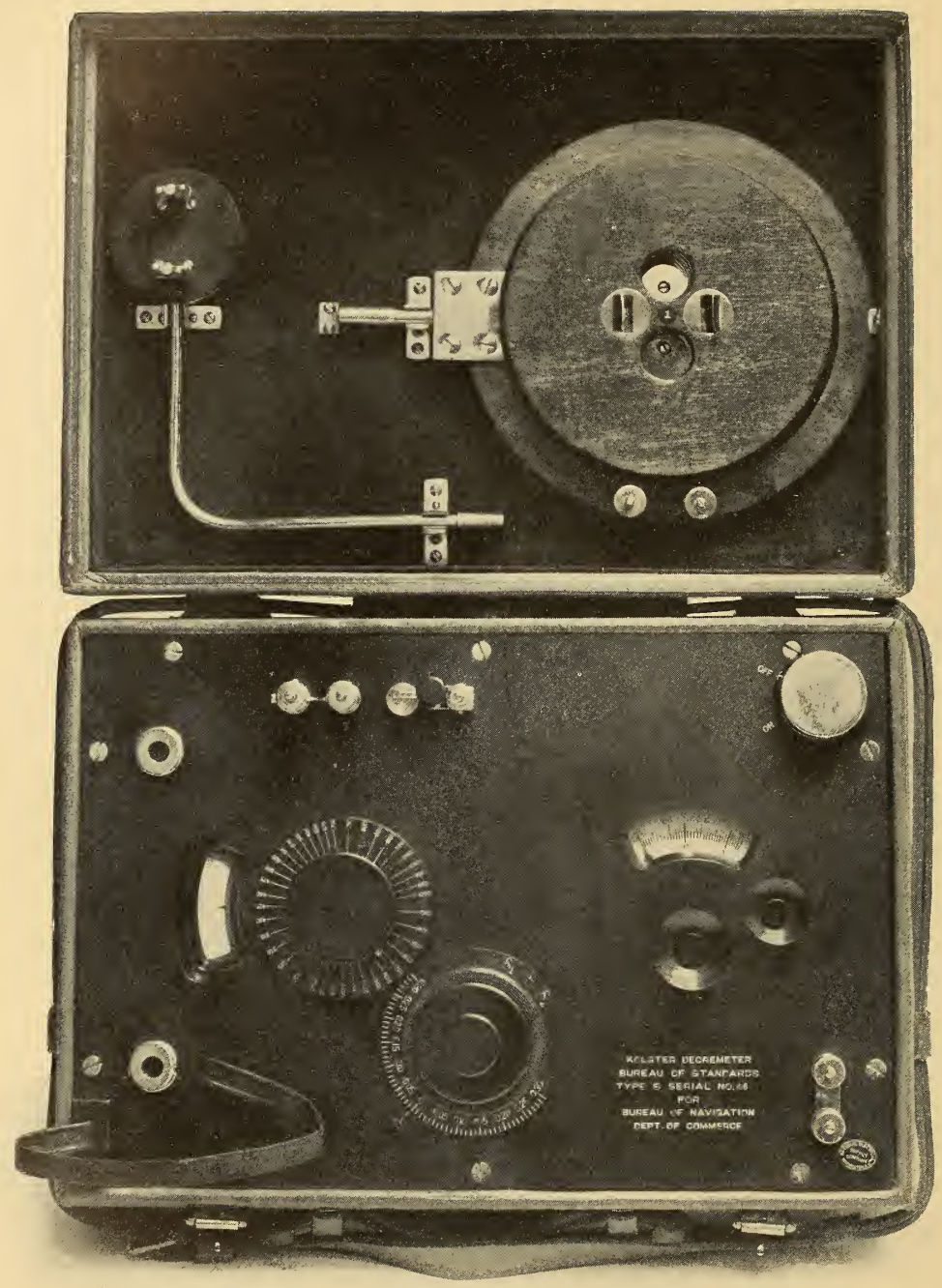

FIG. 2I.-Decremeter mounted in leather carrying case 
In conclusion, I wish to take this opportunity to express my thanks to Mr. E. T. Chamberlain, Commissioner of Navigation, and to Dr. E. B. Rosa, Chief Physicist of the Bureau of Standards, for the keen interest they have taken in the development of the decremeter and for their helpful encouragement which they have given me from time to time.

Washington, August 15, 1914. $82780^{\circ}-15-9$ 